\title{
Serum glucose/potassium ratio as a clinical risk factor for aneurysmal subarachnoid hemorrhage
}

\author{
Yu Fujiki, MD, ${ }^{1}$ Fumihiro Matano, MD, PhD, ${ }^{2}$ Takayuki Mizunari, MD, PhD, ${ }^{2}$ Yasuo Murai, MD, PhD, ${ }^{3}$ \\ Kojiro Tateyama, MD, PhD, ${ }^{3}$ Kenta Koketsu, MD, PhD, ${ }^{3}$ Asami Kubota, MD, ${ }^{2}$ \\ Shiro Kobayashi, MD, PhD, ${ }^{2}$ Hiroyuki Yokota, MD, PhD, ${ }^{1}$ and Akio Morita, MD, PhD ${ }^{3}$
} Departments of ${ }^{1}$ Emergency and Critical Care Medicine and ${ }^{3}$ Neurological Surgery, Nippon Medical School; and ${ }^{2}$ Department of
Neurosurgery, Chiba Hokusoh Hospital, Tokyo, Japan

\begin{abstract}
OBJECTIVE Aneurysmal subarachnoid hemorrhage (SAH) can result in poor outcomes, and biomarkers for predicting poor prognosis have not yet been established. The aim of this study was to clarify the significance of the serum glucosel potassium ratio for predicting the prognosis of aneurysmal SAH.
\end{abstract}

METHODS The authors retrospectively reviewed the records of 565 patients with aneurysmal SAH between 2006 and 2016. The patient group comprised 208 men and 357 women (mean age 61.5 years, range 10-95 years). A statistical analysis was conducted of the clinical and laboratory risk factors of poor outcome, including the serum glucose/potassium ratio.

RESULTS On estimation of the initial assessment using Hunt and Kosnik (H-K) grading, 233 patients (41.2\%) were classified as the severe SAH group (H-K Grade IV or V). There were significant correlations between the severe SAH group and serum glucose/potassium ratio $(p<0.0001)$. Serum glucose/potassium ratio was elevated in an $\mathrm{H}-\mathrm{K}$ gradedependent manner (Spearman's $r=0.5374, p<0.0001$ ). With the estimation of the Glasgow Outcome Scale (GOS) score at discharge, 355 patients (62.8\%) were classified as poor outcome (GOS score 1-3). The serum glucose/potassium ratio was elevated in a GOS score at discharge-dependent manner (Spearman's $r=0.4006, p<0.0001$ ), and was significantly elevated in the poor outcome group compared with the good outcome group (GOS score 4 or $5 ; p=0.0245$ ). There were significant correlations between poor outcome and serum glucose/potassium ratio $(p<0.0001)$, age $(p<$ $0.0001)$, brain natriuretic peptide levels $(p=0.011)$, cerebral infarction due to vasospasm $(p<0.0001)$, and H-K grade ( $p$ $<0.0001)$. Multivariate logistic regression analyses showed significant correlations between poor outcome and serum glucose/potassium ratio $(p=0.009)$.

CONCLUSIONS In this study, the serum glucose/potassium ratio of patients with aneurysmal SAH at admission was significantly correlated with $\mathrm{H}-\mathrm{K}$ grade and GOS score at discharge. Therefore, this ratio was useful for predicting prognosis of aneurysmal SAH, especially in severe cases.

https://thejns.org/doi/abs/10.3171/2017.5.JNS162799

KEY WORDS aneurysm; biomarker; glucose; potassium; subarachnoid hemorrhage; vascular disorders

A NEURYSMAL subarachnoid hemorrhage (SAH) continues to have a poor prognosis despite progress in treatment. ${ }^{8,9}$ The prognosis for aneurysmal SAH has been predicted using Hunt and Kosnik $(\mathrm{H}-\mathrm{K})^{7}$ or World Federation of Neurological Surgeons grading, ${ }^{30}$ which evaluate disturbances of consciousness and neurology. These neurological-based grades cannot be used when sedating drugs have been administered, such as during intubation in resuscitation or preventing reruptured aneurysms in patients with severe SAH status. In addition, despite severe neurological scores, some cases have a good prognosis; therefore, prediction of prognosis using clinical risk factors such as biomarkers with no neurological score are required for decisions of treatment policy, especially in cases of severe aneurysmal SAH. Biomarkers such as C-reactive protein (CRP) ${ }^{6}$ and brain natriuretic peptide (BNP) ${ }^{32}$ were reported to be useful prognostic factors. However, there are few reports on the relationship

ABBREVIATIONS $\mathrm{ACA}=$ anterior cerebral artery; $\mathrm{ACOA}=$ anterior communicating artery; $\mathrm{BA}=$ basilar artery; $\mathrm{BNP}=$ brain natriuretic peptide; $\mathrm{CRP}=\mathrm{C}$-reactive protein; GOS = Glasgow Outcome Scale; $\mathrm{H}-\mathrm{K}=$ Hunt and Kosnik; ICA = internal carotid artery; $\mathrm{MCA}=$ middle cerebral artery; $\mathrm{SAH}=$ subarachnoid hemorrhage; VA = vertebral artery; $\mathrm{WBC}=$ white blood cell.

SUBMITTED November 8, 2016. ACCEPTED May 15, 2017.

INCLUDE WHEN CITING Published online November 17, 2017; DOI: 10.3171/2017.5.JNS162799. 
TABLE 1. Patient characteristics

\begin{tabular}{|c|c|}
\hline Variable & Value \\
\hline No. of patients & 565 \\
\hline Males/females & $208 / 357$ \\
\hline Mean age in yrs (range) & $61.5(10-95)$ \\
\hline \multicolumn{2}{|l|}{ H-K Grade } \\
\hline I & 48 \\
\hline II & 186 \\
\hline III & 98 \\
\hline IV & 89 \\
\hline V & 144 \\
\hline \multicolumn{2}{|l|}{ Fisher grade } \\
\hline 1 & 20 \\
\hline 2 & 18 \\
\hline 3 & 513 \\
\hline 4 & 14 \\
\hline Mean aneurysm size in $\mathrm{mm}$ (range) & $7.1(1.8-31)$ \\
\hline \multicolumn{2}{|l|}{ Aneurysm location } \\
\hline ACoA/ACA & 158 \\
\hline ICA & 136 \\
\hline MCA & 97 \\
\hline BA & 30 \\
\hline VA & 44 \\
\hline Others & 100 \\
\hline \multicolumn{2}{|l|}{ GOS score at discharge } \\
\hline 5 & 154 \\
\hline 4 & 56 \\
\hline 3 & 165 \\
\hline 2 & 58 \\
\hline 1 & 132 \\
\hline
\end{tabular}

Data expressed as number of patients unless otherwise indicated.

between serum glucose/potassium ratio and prognosis of aneurysmal SAH. We analyzed this relationship in the present study.

\section{Methods}

The study is reported based on criteria from the STROBE (Strengthening the Reporting of Observational Studies in Epidemiology) statement. The study was approved by Chiba Hokusoh Hospital Research Ethics Committee in Chiba, Japan.

\section{Patient Characteristics}

Patient characteristics, SAH grading, Fisher grade, aneurysmal size and location, and outcomes are shown in Table 1 . We treated 565 patients with aneurysmal SAH at our hospital between 2006 and 2016.

The patient group comprised 208 men and 357 women, and the mean patient age was 61.5 years (range 10-95 years). H-K grading was as follows: Grade I, 48 cases; Grade II, 186 cases; Grade III, 98 cases; Grade IV, 89 cases; and Grade V, 144 cases. Aneurysms ranged in size from 1.8 to $31 \mathrm{~mm}$ (mean $7.1 \mathrm{~mm}$ ), and the locations were as follows: anterior communicating artery (ACoA) or anterior cerebral artery (ACA), 158 cases; internal carotid artery (ICA), 136 cases; middle cerebral artery (MCA), 97 cases; basilar artery (BA), 30 cases; and vertebral artery (VA), 44 cases. Among 565 cases, 161 (29\%) were transferred to our hospital from another hospital. The time from $\mathrm{SAH}$ to admission ranged from 1 hour to 16 days (mean time $20.1 \pm 19.07$ hours). We retrospectively reviewed data from patients' charts, including admission data, laboratory workup, imaging studies, and follow-up reports.

\section{Preoperative Examination and Treatment}

We diagnosed SAH by CT on the day of admission. Clinical severities were estimated in all patients by a neurosurgeon at first contact, using $\mathrm{H}-\mathrm{K}$ grading. We defined severe $\mathrm{SAH}$ as $\mathrm{H}-\mathrm{K}$ Grades IV or $\mathrm{V}$ and nonsevere SAH as H-K Grades I-III. We investigated laboratory data on admission at our hospital, including potassium, glucose, white blood cell (WBC), CRP, and BNP levels. All patients underwent cerebral angiography to determine the ruptured aneurysm size and location. At our institution, we treat aneurysmal SAH by clip placement in the acute stage ( $<2$ days). Perioperative care was performed according to a standardized protocol provided by the guidelines for the management of aneurysmal SAH in Japan. ${ }^{3}$ All patients underwent angiography on postoperative Day 7 to detect the cerebral vasospasm and confirm complete clip ligation. We checked glucose levels 4 times per day and managed to control the blood glucose value postoperatively at $144-180 \mathrm{mg} / \mathrm{dl}$ using sliding-scale insulin. ${ }^{5}$

\section{Outcome Assessment}

Patient prognosis was evaluated at the time of discharge and 3 months after discharge using the Glasgow Outcome Scale (GOS). ${ }^{2}$ Good outcome was defined as good recovery (score of 5) or moderate disability (score of 4), and poor outcome was defined as severe disability (score of 3 ), vegetative state (score of 2), or death (score of 1 ).

Cerebral vasospasm was judged by more than 2 neurosurgeons and was defined as less than $50 \%$ of the narrowing vessel diameter in the $\mathrm{M}_{1}, \mathrm{M}_{2}, \mathrm{~A}_{1}$, and $\mathrm{P}_{1}$ portions of the arteries compared with initial values, and complicated cerebral infarction was judged by more than 2 neurosurgeons using MR diffusion-weighted imaging as the appearance of clinical symptoms within Day 3-14, such as loss of consciousness or newly developed focal neurological deficits, including aphasia, hemiparesis, and disturbance of consciousness.

\section{Statistical Analysis}

Statistical analysis was performed using SPSS for Mac (version 21.0, IBM Corp.). Variables are expressed as mean \pm SD or number of patients (percentage), where appropriate. The relationship between severe SAH (H-K Grade IV or V), poor outcome (GOS poor outcome at discharge), and these parameters was investigated. The normality of the data was evaluated using the ShapiroWilk test. The normally distributed continuous variables were compared using the Student t-test and nonnormally 
TABLE 2. The differences in imaging and laboratory data between severe and nonsevere SAH

\begin{tabular}{lcccc}
\hline \multicolumn{1}{c}{ Variable } & Total & Nonsevere SAH & Severe SAH† & $p$ Value \\
\hline Mean age in yrs (SD) & $61.5(14.7)$ & $58.8(14.4)$ & $65.3(14.3)$ & $<0.0001$ \\
\hline Males, no. (\%) & $208(36.8)$ & $132(39.7)$ & $76(32.7)$ & 0.2093 \\
\hline Aneurysm size in mm (SD) & $7.09(4.50)$ & $6.35(3.56)$ & $8.18(5.44)$ & 0.0115 \\
\hline Fisher Grade 3, no. (\%) & $513(90.8)$ & $291(87.6)$ & $222(95.2)$ & $\mathbf{0 . 0 0 1 8}$ \\
\hline Glucose/potassium ratio (SD) & $52.1(21.1)$ & $43.8(14.8)$ & $63.9(23.1)$ & $<0.0001$ \\
\hline WBC count (SD) & $11,617(4565)$ & $10,933(3853)$ & $12,597(5279)$ & $\mathbf{0 . 0 0 0 5}$ \\
\hline CRP in mg/L (SD) & $0.61(2.04)$ & $0.49(1.43)$ & $0.77(2.68)$ & 0.0831 \\
\hline BNP in pg/ml (SD) & $267(653)$ & $264(843)$ & $279(302)$ & $\mathbf{0 . 0 1 7 6 7}$ \\
\hline
\end{tabular}

Variables showing significant difference according to univariate analysis $(p<0.05)$ are indicated by boldface type.

* H-K Grade I-III ( $n=332)$.

$\dagger \mathrm{H}-\mathrm{K}$ Grade IV or V $(n=233)$.

distributed variables using the Mann-Whitney U-test. The correlations between H-K grade and serum glucose/potassium ratio were analyzed using Spearman's rank correlation coefficient. The correlations between GOS score at discharge and serum glucose/potassium ratio were also analyzed using Spearman's rank correlation coefficient. Comparisons in glucose/potassium ratio between good outcome (GOS score 4 or 5) and poor outcome (GOS score 1-3) were examined using Mann-Whitney U-tests. Multivariate logistic regression analysis was performed for all variables, including age, sex, aneurysm size, glucose/potassium ratio, glucose, potassium, WBC count, CRP level, and BNP level, for the purpose of identifying the relationship between variables that were significantly associated with poor outcome (GOS poor outcome at discharge). Differences were considered significant at a $\mathrm{p}$ value $<0.05$.

\section{Results}

With the estimation of the initial assessment of $\mathrm{H}-\mathrm{K}$ grading, 233 patients (41.2\%) were classified as the severe

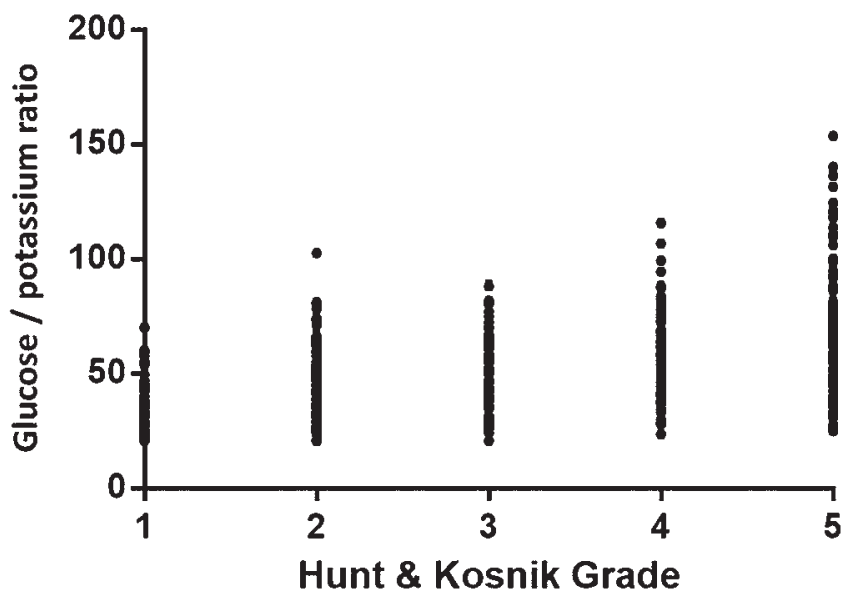

FIG. 1. Scatterplot showing the serum glucose/potassium ratio increased in an H-K grade-dependent manner (Spearman's $r=0.5374$, $p<0.0001)$.
SAH group (H-K Grade IV or V; Table 1). There were significant correlations between the severe $\mathrm{SAH}$ group and age $(\mathrm{p}<0.0001)$, aneurysm size $(\mathrm{p}=0.0115)$, Fisher Grade $3(p=0.0018)$, serum glucose/potassium ratio $(p<0.0001)$, WBC count $(\mathrm{p}=0.0005)$, and BNP level $(\mathrm{p}=0.01767$; Table 2). Serum glucose/potassium ratio was elevated in an H-K grade-dependent manner (Spearman's $r=0.5374$, p $<0.0001$; Fig. 1).

With the estimation of the GOS score at discharge, 355 patients $(62.8 \%)$ were classified as poor outcome (GOS score 1-3). With the estimation of GOS score at 3 months after discharge, 308 patients $(54.5 \%)$ were classified as poor outcome (GOS score 1-3). Serum glucose/potassium ratio was elevated in a GOS score at discharge-dependent manner (Spearman's $r=0.4006, p<0.0001$; Fig. 2), and was significantly elevated in the poor outcome group compared with the good outcome group $(\mathrm{p}=0.0245$; Fig. 3$)$.

There were significant correlations between poor outcome (GOS score 1-3) and age ( $p<0.0001)$, serum glucose/potassium ratio $(p<0.0001)$, glucose $(p<0.0001)$, potassium $(\mathrm{p}=0.0001), \mathrm{BNP}(\mathrm{p}=0.011)$, cerebral infarc-

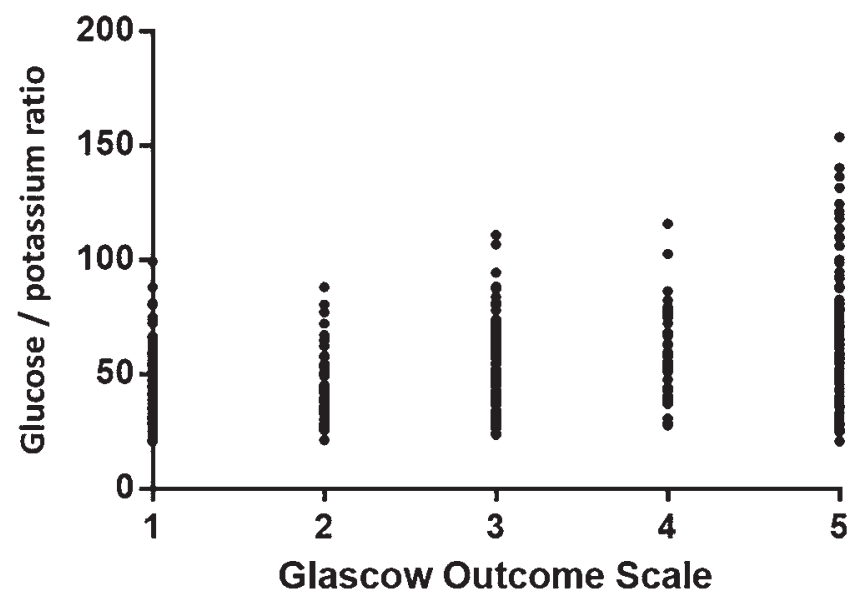

FIG. 2. Scatterplot showing the serum glucose/potassium ratio increased in a GOS score-dependent manner (Spearman's $r=0.4006$, $p<0.0001)$. 


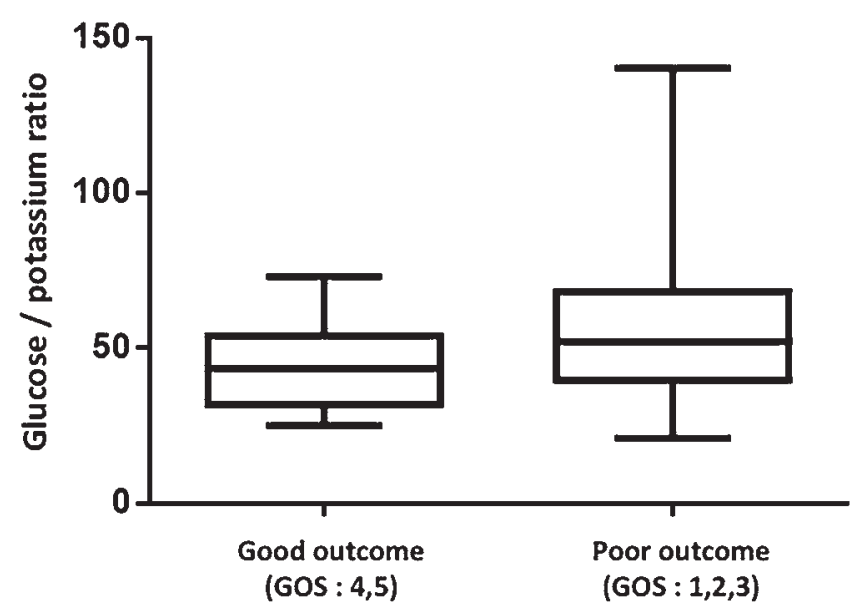

FIG. 3. Box-and-whiskers plot demonstrating that in the H-K Grade IV or $\checkmark$ group, the serum glucose/potassium ratio is significantly higher in the GOS poor outcome group than in the good outcome group $(p=0.0245)$.

tion due to vasospasm ( $\mathrm{p}<0.0001)$, and $\mathrm{H}-\mathrm{K}$ grade ( $\mathrm{p}<$ 0.0001; Table 3). Multivariate logistic regression analyses showed significant correlations between poor outcome (GOS score 1-3) and serum glucose/potassium ratio ( $\mathrm{p}=$ $0.009)$, glucose $(p=0.05)$, potassium $(p=0.023)$, and cerebral infarction due to vasospasm ( $\mathrm{p}=0.01$; Table 4$)$.

\section{Discussion}

In the present study, the serum glucose/potassium ratio in cases of aneurysmal SAH was significantly associated with $\mathrm{H}-\mathrm{K}$ grade and GOS score at discharge. In addition, the glucose/potassium ratio in the severe $\mathrm{SAH}$ group $(\mathrm{H}-\mathrm{K}$ Grade 4 or 5) was significantly elevated in the poor outcome group (GOS score 1-3) compared with the good outcome group (GOS score 4 or 5). Therefore, the serum glucose/potassium ratio was useful for prediction of outcome, especially in the severe SAH group.

Aneurysmal SAH remains a challenging disease with high rates of mortality and morbidity, despite improved treatment to date. ${ }^{16}$ In particular, patients with severe aneurysmal SAH evaluated by H-K or World Federation of Neurological Surgeons grading tend to have poor outcomes, with those staying longer in the hospital having a high risk of dying during admission. ${ }^{33}$ However, some patients with severe aneurysmal SAH have a good prognosis. 22,29 Therefore, decisions regarding treatment policy, especially in cases with severe aneurysmal $\mathrm{SAH}$, are extremely important, not only from the viewpoint of patient outcome but also medical economy. ${ }^{14,25}$ Excessive catecholamine secretion, such as epinephrine and norepinephrine, occurs in the majority of aneurysmal SAH cases, causing sympathetic nervous system activation especially in severe cases, ${ }^{12,19,20}$ resulting in the occurrence of systemic complications such as pulmonary cardiac lung edema, arrhythmia, or heart failure, ${ }^{11,21,28}$ and reflect the primary brain injury itself. Yokobori et al ${ }^{32}$ reported that serum BNP was significantly correlated with SAH grading and was a useful biomarker of systemic clinical impact, reflecting increased cardiac preload and afterload in severe aneurysmal SAH. Because of the breakdown of the blood-brain barrier by the ruptured aneurysm, increasing leukocyte trafficking has also been reported. ${ }^{4}$ Inflammation biomarkers such as $\mathrm{CRP}^{6}$ or tumor necrosis factor- $\alpha^{27}$ transport the blood clots of the subarachnoid space to the blood because of immediate systemic inflammatory response. Some previous reports have also shown the relationships between several biomarkers and severe grading of aneurysmal SAH. ${ }^{23,26}$ However, these tests have not been widely used in various facilities in the clinical setting because of the lack of feasibility until now. Serum glucose values have correlated with patient clinical condition, and the role of the serum glucose level as

TABLE 3. Results of statistical analysis of the risk factors for poor outcome in aneurysmal SAH

\begin{tabular}{|c|c|c|c|c|}
\hline Variable & Total & Good Outcome* & Poor Outcome† & p Value \\
\hline Mean age in yrs (SD) & $61.5(14.7)$ & $53.8(12.8)$ & $66.1(13.9)$ & $<0.0001$ \\
\hline Males & $208(36.8)$ & $86(40.9)$ & $122(34.3)$ & 0.1254 \\
\hline Posterior circulation aneurysm location & $96(17)$ & $39(18.6)$ & $57(16.1)$ & 0.4870 \\
\hline Aneurysm size in mm (SD) & $7.09(4.5)$ & $6.31(3.51)$ & $7.61(4.99)$ & 0.067 \\
\hline Fisher Grade 3 & $513(90.8)$ & $179(85.2)$ & $334(94.1)$ & 0.0008 \\
\hline Glucose/potassium ratio (SD) & $52.1(21.1)$ & $42.3(15.1)$ & $57.9(22)$ & $<0.0001$ \\
\hline Glucose in mg/dl (SD) & $182(63.9)$ & $153(44.3)$ & $200(65.5)$ & $<0.0001$ \\
\hline Potassium in mmol/L (SD) & $3.6(0.51)$ & $3.7(0.46)$ & $3.6(0.53)$ & 0.0001 \\
\hline WBC count (SD) & $11,827(6812)$ & $10,871(3974)$ & $12,389(7976)$ & 0.005 \\
\hline CRP (SD) & $0.61(2.04)$ & $0.32(0.67)$ & $0.77(2.5)$ & 0.7989 \\
\hline BNP (SD) & $204(258)$ & $104(133)$ & $244(285)$ & 0.011 \\
\hline Cerebral infarction due to vasospasm & $50(8.8)$ & $3(1.5)$ & 47 (13.2) & $<0.0001$ \\
\hline H-K severe Grade IV or V & $233(41.2)$ & $18(8.6)$ & $215(60.6)$ & $<0.0001$ \\
\hline
\end{tabular}


TABLE 4. Multivariate logistic regression analysis for risk factors of poor outcome in aneurysmal SAH

\begin{tabular}{lcl}
\hline \multicolumn{1}{c}{ Variable } & OR $(95 \% \mathrm{Cl})$ & $\mathrm{p}$ Value \\
\hline Age $>60$ & $0.73(0.4-1.2)$ & 0.281 \\
\hline Glucose/potassium ratio $>60$ & $13(0.98-184)$ & 0.05 \\
\hline Cerebral infarction due to vasospasm & $16(3.6-77)$ & $\mathbf{0 . 0 1}$ \\
\hline Glucose/potassium ratio & & $\mathbf{0 . 0 0 9}$ \\
\hline Glucose & & 0.05 \\
\hline Potassium & & $\mathbf{0 . 0 2 3}$ \\
\hline
\end{tabular}

Variables showing significant difference according to univariate analysis $(p<$ 0.05 ) are indicated by boldface type.

a predictor for poor outcome after aneurysmal SAH has been reported. ${ }^{10,15,17}$

Juvela et al ${ }^{10}$ reported that the initial serum glucose level at admission was independently associated with the severity of aneurysmal SAH, and these poor outcomes were without a relationship to serum glucose level and frequency of occurrence for cerebral vasospasm, or permanent cerebral ischemia. Catecholamines, glucagon, and corticosteroids are primary glycol-regulatory hormones implicated in the hyperglycemic reaction., ${ }^{1,31}$ Particularly important after injury and stress are catecholamines because they increase glucose levels directly and indirectly by increasing glucagon secretion and inhibiting insulin secretion. . $^{13,15}$

Potassium in the human body is mostly stored within the cells, and its transportation is controlled by active cellular uptake of potassium through the cell membrane and adenosine triphosphatase sodium/potassium pump $\left(\mathrm{Na}^{+} /\right.$ $\mathrm{K}^{+}$-ATPase). Catecholamines, $\beta 2$ adrenergic hormones, and insulin regulate $\mathrm{Na}^{+} / \mathrm{K}^{+}$-ATPase and results in a decrease in the serum potassium level. ${ }^{18,24}$ Reid et al. ${ }^{24}$ reported epinephrine-induced hypokalemia results from stimulation of a beta-adrenoceptor linked to membrane $\mathrm{Na} / \mathrm{K}$-ATPase causing potassium influx in vivo. In addition, aneurysmal SAH has a high serum glucose value by excessive catecholamine secretion as mentioned above; therefore, this situation promotes the secretion of insulin and serum potassium entry into the cells.

In the present study, the serum glucose/potassium ratio was significantly associated with $\mathrm{H}-\mathrm{K}$ grading and GOS score at discharge; therefore, the serum glucose/potassium ratio is reflective of excessive catecholamine due to aneurysmal SAH, and may indicate preoperative severity and postoperative prognosis. Although serum glucose and potassium have been exclusively related to SAH severity and prognosis, a stronger correlation was detected by obtaining the ratio on multivariate logistic regression analysis. In addition, the serum glucose/potassium ratio was significantly higher in the poor outcome group among patients with severe $\mathrm{SAH}$, and measurement of these levels can be easily undertaken in any institution. Therefore, the serum glucose/potassium ratio may serve to aid in the decision regarding surgical indication in severe SAH in the future.

\section{Limitations of the Study}

This study had several limitations. First, we did not investigate serum endocrine levels such as catecholamine, glucagon, and corticosteroids; therefore, the real cause of the high level of serum glucose/potassium ratio in severe aneurysmal SAH was not clear. Second, we investigated patient prognosis only at discharge and 3 months after discharge; therefore, a long-term follow-up study is needed in the future. And third, our analysis was retrospective and conducted at a single center. A prospective and multicenter trial is needed to support our claim in the future.

\section{Conclusions}

In this paper we discussed and demonstrated the utility of the serum glucose/potassium ratio in cases of aneurysmal SAH. The serum glucose/potassium ratio was useful for assessing the status and predicting the prognosis of aneurysmal SAH, and it may serve as an aid in the decision regarding surgical indication in severe $\mathrm{SAH}$ in the future.

\section{References}

1. Bessey PQ, Watters JM, Aoki TT, Wilmore DW: Combined hormonal infusion simulates the metabolic response to injury. Ann Surg 200:264-281, 1984

2. Bond MR, Jennett WB, Brooks DN, McKinlay W: The nature of physical, mental and social deficits contributing to the categories of good recovery, moderate and severe disability in the Glasgow Global Outcome Scale. Acta Neurochir Suppl (Wien) 28:126-127, 1979

3. Committee for Guidelines for Management of Aneurysmal Subarachnoid Hemorrhage, Japanese Society on Surgery for Cerebral Stroke: Evidence-based guidelines for the management of aneurysmal subarachnoid hemorrhage. English Edition. Neurol Med Chir (Tokyo) 52:355-429, 2012

4. Dóczi T, Joó F, Adám G, Bozóky B, Szerdahelyi P: Bloodbrain barrier damage during the acute stage of subarachnoid hemorrhage, as exemplified by a new animal model. Neurosurgery 18:733-739, 1986

5. Finfer S, Chittock DR, Su SY, Blair D, Foster D, Dhingra V, et al: Intensive versus conventional glucose control in critically ill patients. N Engl J Med 360:1283-1297, 2009

6. Fountas KN, Tasiou A, Kapsalaki EZ, Paterakis KN, Grigorian AA, Lee GP, et al: Serum and cerebrospinal fluid Creactive protein levels as predictors of vasospasm in aneurysmal subarachnoid hemorrhage. Clinical article. Neurosurg Focus 26(5):E22, 2009

7. Hunt WE, Kosnik EJ: Timing and perioperative care in intracranial aneurysm surgery. Clin Neurosurg 21:79-89, 1974

8. Inagawa T: Trends in incidence and case fatality rates of aneurysmal subarachnoid hemorrhage in Izumo City, Japan, between 1980-1989 and 1990-1998. Stroke 32:1499-1507, 2001

9. Juvela S: Prehemorrhage risk factors for fatal intracranial aneurysm rupture. Stroke 34:1852-1857, 2003

10. Juvela S, Siironen J, Kuhmonen J: Hyperglycemia, excess weight, and history of hypertension as risk factors for poor outcome and cerebral infarction after aneurysmal subarachnoid hemorrhage. J Neurosurg 102:998-1003, 2005

11. Koenig MA, Puttgen HA, Prabhakaran V, Reich D, Stevens RD: B-type natriuretic peptide as a marker for heart failure in patients with acute stroke. Intensive Care Med 33:15871593, 2007

12. Kuroda Y: Neurocritical care update. J Intensive Care 4:36, 2016

13. Kurtz P, Claassen J, Schmidt JM, Helbok R, Hanafy KA, 
Presciutti M, et al: Reduced brain/serum glucose ratios predict cerebral metabolic distress and mortality after severe brain injury. Neurocrit Care 19:311-319, 2013

14. Lai PM, Dasenbrock H, Lin N, Du R: The impact of insurance status on the outcomes after aneurysmal subarachnoid hemorrhage. PLoS One 8:e78047, 2013

15. Lanzino G, Kassell NF, Germanson T, Truskowski L, Alves W: Plasma glucose levels and outcome after aneurysmal subarachnoid hemorrhage. J Neurosurg 79:885-891, 1993

16. Le Roux PD, Elliott JP, Newell DW, Grady MS, Winn HR: Predicting outcome in poor-grade patients with subarachnoid hemorrhage: a retrospective review of 159 aggressively managed cases. J Neurosurg 85:39-49, 1996

17. Leijenaar JF, Dorhout Mees SM, Algra A, van den Bergh WM, Rinkel GJ: Effect of magnesium treatment and glucose levels on delayed cerebral ischemia in patients with subarachnoid hemorrhage: a substudy of the Magnesium in Aneurysmal Subarachnoid Haemorrhage trial (MASH-II). Int J Stroke 10 (Suppl A100):108-112, 2015

18. Massara F, Tripodina A, Rotunno M: Propranolol block of epinephrine-induced hypokaliaemia in man. Eur J Pharmacol 10:404-407, 1970

19. Naredi S, Lambert G, Edén E, Zäll S, Runnerstam M, Rydenhag B, et al: Increased sympathetic nervous activity in patients with nontraumatic subarachnoid hemorrhage. Stroke 31:901-906, 2000

20. Nyberg C, Karlsson T, Hillered L, Stridsberg M, Ronne Engström E: The early endocrine stress response in experimental subarachnoid hemorrhage. PLoS One 11:e0151457, 2016

21. Nyberg C, Karlsson T, Ronne-Engström E: Predictors of increased cumulative serum levels of the $\mathrm{N}$-terminal prohormone of brain natriuretic peptide 4 days after acute spontaneous subarachnoid hemorrhage. J Neurosurg 120:599-604, 2014

22. Pegoli M, Mandrekar J, Rabinstein AA, Lanzino G: Predictors of excellent functional outcome in aneurysmal subarachnoid hemorrhage. J Neurosurg 122:414-418, 2015

23. Petzold A, Keir G, Kerr M, Kay A, Kitchen N, Smith M, et al: Early identification of secondary brain damage in subarachnoid hemorrhage: a role for glial fibrillary acidic protein. J Neurotrauma 23:1179-1184, 2006

24. Reid JL, Whyte KF, Struthers AD: Epinephrine-induced hypokalemia: the role of beta adrenoceptors. Am J Cardiol 57:23F-27F, 1986

25. Sakhuja A, Schold JD, Kumar G, Katzan I, Navaneethan SD: Nontraumatic subarachnoid hemorrhage in maintenance dialysis hospitalizations: trends and outcomes. Stroke 45:71-76, 2014

26. Shim JH, Yoon SM, Bae HG, Yun IG, Shim JJ, Lee KS, et al: Which treatment modality is more injurious to the brain in patients with subarachnoid hemorrhage? Degree of brain damage assessed by serum S100 protein after aneurysm clipping or coiling. Cerebrovasc Dis 34:38-47, 2012

27. Starke RM, Chalouhi N, Jabbour PM, Tjoumakaris SI, Gonzalez LF, Rosenwasser RH, et al: Critical role of TNF- $\alpha$ in cerebral aneurysm formation and progression to rupture. J Neuroinflammation 11:77, 2014

28. Sviri GE, Feinsod M, Soustiel JF: Brain natriuretic peptide and cerebral vasospasm in subarachnoid hemorrhage. Clinical and TCD correlations. Stroke 31:118-122, 2000

29. Taylor CJ, Robertson F, Brealey D, O'shea F, Stephen T, Brew $\mathrm{S}$, et al: Outcome in poor grade subarachnoid hemorrhage patients treated with acute endovascular coiling of aneurysms and aggressive intensive care. Neurocrit Care 14:341-347, 2011

30. Teasdale GM, Drake CG, Hunt W, Kassell N, Sano K, Pertuiset B, et al: A universal subarachnoid hemorrhage scale: report of a committee of the World Federation of Neurosurgical Societies. J Neurol Neurosurg Psychiatry 51:1457, 1988

31. van Donkelaar CE, Dijkland SA, van den Bergh WM, Bakker J, Dippel DW, Nijsten MW, et al: Early circulating lactate and glucose levels after aneurysmal subarachnoid hemorrhage correlate with poor outcome and delayed cerebral ischemia: a two-center cohort study. Crit Care Med 44:966-972, 2016

32. Yokobori S, Watanabe A, Igarashi Y, Tagami T, Kuwamoto $\mathrm{K}$, Ishinokami S, et al: The serum level of brain natriuretic peptide increases in severe subarachnoid hemorrhage thereby reflecting an increase in both cardiac preload and afterload. Cerebrovasc Dis 38:276-283, 2014

33. Zhao B, Yang H, Zheng K, Li Z, Xiong Y, Tan X, et al: Preoperative and postoperative predictors of long-term outcome after endovascular treatment of poor-grade aneurysmal subarachnoid hemorrhage. J Neurosurg 126:1764-1771, 2016

\section{Disclosures}

The authors report no conflict of interest concerning the materials or methods used in this study or the findings specified in this paper.

\section{Author Contributions}

Conception and design: Fujiki, Matano. Acquisition of data: Fujiki. Analysis and interpretation of data: Fujiki, Matano. Drafting the article: Fujiki, Matano, Yokota. Critically revising the article: Mizunari, Murai, Tateyama, Koketsu, Kubota, Kobayashi, Yokota, Morita. Reviewed submitted version of manuscript: Mizunari, Murai, Tateyama, Koketsu, Kubota, Kobayashi, Morita. Approved the final version of the manuscript on behalf of all authors: Fujiki. Statistical analysis: Fujiki, Matano. Administrative/technical/ material support: Mizunari, Murai, Tateyama, Koketsu, Kubota, Kobayashi, Yokota, Morita. Study supervision: Mizunari, Murai, Tateyama, Koketsu, Kubota, Kobayashi, Yokota, Morita.

\section{Correspondence}

Yu Fujiki, Department of Emergency and Critical Care Medicine, Nippon Medical School, 1-1-5 Sendagi, Bunkyo-ku, Tokyo 1138603, Japan. email: hawkswords51@gmail.com. 